\title{
Sensitivity and specificity of combination of Hull airway reflux questionnaire and gastroesophageal reflux disease questionnaire in identifying patients with gastroesophageal reflux-induced chronic cough
}

\author{
Siwan Wen", Shengyuan Wang", Shanshan Niu, Mengru Zhang, Cuiqin Shi, Zhongmin Qiu, \\ Xianghuai Xu, Li Yu \\ Department of Pulmonary and Critical Care Medicine, Tongji Hospital, Tongji University, School of Medicine, Shanghai, China \\ Contributions: (I) Conception and design: S Wen, S Wang, X Xu, L Yu; (II) Administrative support: L Yu; (III) Provision of study materials or patients: \\ All authors; (IV) Collection and assembly of data: All authors; (V) Data analysis and interpretation: S Wen, S Wang; (VI) Manuscript writing: All \\ authors; (VII) Final approval of manuscript: All authors. \\ "These authors contributed equally to this work. \\ Correspondence to: Xianghuai Xu; Li Yu. Department of Pulmonary and Critical Care Medicine, Tongji Hospital, Tongji University, School of \\ Medicine, 389 Xincun Road, Shanghai, China. Email: 05849@tongji.edu.cn; yuli0219@sina.com.
}

\begin{abstract}
Background: There is a need to find a simple, non-invasive and effective diagnostic tool for diagnosing gastroesophageal reflux-induced chronic cough (GERC) in clinic. This study aimed to evaluate the predictive diagnostic value of Hull airway reflux questionnaire (HARQ) and its combination with gastroesophageal reflux disease questionnaire (GerdQ) for GERC.

Methods: Chronic cough patients were enrolled and the diagnosis of GERC was established according to the chronic cough diagnosis and treatment process. The diagnostic value of HARQ and GerdQ alone or the combination of HARQ and GerdQ was analyzed.

Results: A total of 402 patients with chronic cough were eventually enrolled, including 166 GERC patients. When the HARQ score was used to predict the diagnosis of GERC, the area under the ROC curve was 0.796 . The sensitivity and specificity were $77.19 \%$ and $77.06 \%$, respectively. When the GerdQ was used to predict the diagnosis of GERC, the area under the ROC curve was 0.763 . The sensitivity and specificity were $70.18 \%$ and $76.15 \%$, respectively. When HARQ combined with GerdQ were used to predict the diagnosis of GERC, the area under the ROC curve was 0.848 . The sensitivity and specificity were $77.19 \%$ and $79.82 \%$, respectively.

Conclusions: HARQ used to evaluate the cough hypersensitivity has a certain predictive diagnostic value for GERC. The diagnosis of GERC should be considered when the HARQ score is $\geq 24$. The predictive diagnostic value of the combination of HARQ and GerdQ is significantly higher, which makes the diagnosis of GERC simpler, quicker and more effective.
\end{abstract}

Keywords: Hull airway reflux questionnaire (HARQ); gastroesophageal reflux disease questionnaire (GerdQ); gastroesophageal reflux-induced chronic cough (GERC); predictive diagnosis

Submitted Apr 09, 2020. Accepted for publication Sep 25, 2020.

doi: $10.21037 /$ atm-20-3236

View this article at: http://dx.doi.org/10.21037/atm-20-3236 


\section{Introduction}

Gastroesophageal reflux-induced chronic cough (GERC) is a gastroesophageal reflux disease characterized by chronic cough, which is one of the common causes of chronic cough (1-3). Chronic cough patients usually have a long disease response time, a long cough relief time, and a low quality of life (4). At present, cough guidelines in various countries recommend empirical anti-reflux therapy as an important method for diagnosing GERC, while the sensitivity and specificity are low. Multichannel intraluminal esophageal impedance and $\mathrm{pH}$ monitoring (MII-pH) is an important method for diagnosing GERC, which has not yet been universally adopted in clinic due to its invasiveness and patients' intolerance to it. The diagnostic criteria have not been unified yet. Besides, the sensitivity of gastroscopy and barium meal examination is also low. Thus, there is a need to find a simple, non-invasive and effective diagnostic tool for GERC. Our previous research found that a gastroesophageal reflux disease questionnaire (GerdQ) value greater than or equal to 8 points could assist the diagnosis of acid GERC, while the evaluation value of non-acid GERC was limited; and GerdQ lacks specific options to evaluate cough (5). We are always devoting ourselves to look for a more comprehensive and effective tool for diagnosing GERC. Hull airway reflux questionnaire (HARQ) was proposed to evaluate the cough hypersensitivity by Morice in 2011 (6). In our previous studies, it was found that the HARQ score of GERC patients was higher than chronic cough patients with other etiologies (7). Therefore, we speculated that HARQ alone or in combination with GerdQ may be suitable for the simple and effective diagnosis of GERC. We then conducted a prospective clinical trial to assess the diagnostic value and limitations of HARQ and its combination with GerdQ for GERC. We present the following article in accordance with the STARD reporting checklist (available at http://dx.doi. org/10.21037/atm-20-3236).

\section{Methods}

\section{Patients}

Study recruitment was guided by an expected $12 \%$ prevalence of chronic cough and a point estimate of $80 \%$ sensitivity. We planned to recruit approximately 400 participants. Patients with chronic cough who visited our department from January 2015 through July 2019 were included. Inclusion criteria were as follows: (I) 16 years old $\leq$ age $\leq 80$ years old; (II) cough course $>8$ weeks; (III) forced expiratory volume in 1 second/forced vital capacity $\left(\mathrm{FEV}_{1} / \mathrm{FVC}\right)>70 \%$, and percentage of predicted $\mathrm{FEV}_{1}$ value $>80 \%$ of the expected value; (IV) those who can understand and complete the contents of HARQ and GerdQ correctly. Exclusion criteria were as follows: (I) pregnant and lactating women; (II) people who smoke or quit smoking within 2 years; (III) people with reading and writing disabilities; (IV) people who have received treatments targeting the cause of cough with improved symptoms; (V) patients with any symptom like wheezing, hemoptysis, or fever; (VI) patients with rales upon lung physical examination; (VII) patients with obvious abnormalities in chest X-ray or chest CT images. Drop-out criteria were as follows: (I) patients who lost to visit; (II) patients whose information was incomplete. The study was conducted in accordance with the Declaration of Helsinki (as revised in 2013). The study was approved by the Ethics Committee of our Hospital (No. K-2015-007) and registered in the Chinese Clinical Trials Register (http://www.chictr.org.cn/) (ChiCTRDDD-17012869). Informed consent was taken from all the patients.

\section{Diagnostic criteria of GERC}

Patients who met the following criteria were diagnosed with GERC (8): (I) mainly daytime cough, (II) abnormal acid or non-acid reflux confirmed by MII-pH. Abnormal gastroesophageal reflux was defined when the DeMeester score was $\geq 12.70$ and/or syndrome association probability (SAP) was $\geq 80 \%$ for abnormal acid GERC or when the SAP was $\geq 80 \%$ for abnormal non-acid GERC (9). (III) Cough responsive to a stepwise anti-reflux therapy (cough symptom score decreased by $>50 \%$ ) (10). A standard anti-reflux therapy (omeprazole $20 \mathrm{mg}$ twice daily plus mosapride $10 \mathrm{mg}$ thrice daily) was first introduced to suspected GERC patients. If no remission of cough was achieved, the dose of proton pump inhibitor was doubled (omeprazole $40 \mathrm{mg}$ twice daily) and continued for 8 weeks. Patients who responded to the double-dosing were kept on this treatment until their cough resolved. If the cough was not resolved, then baclofen was introduced (omeprazole $20 \mathrm{mg}$ twice daily, baclofen $10-20 \mathrm{mg}$ thrice daily) for 4 weeks, while mosapride was discontinued. After that, the remaining non-responders were escalated to omeprazole (20 mg twice daily) with gabapentin (100-300 mg thrice 


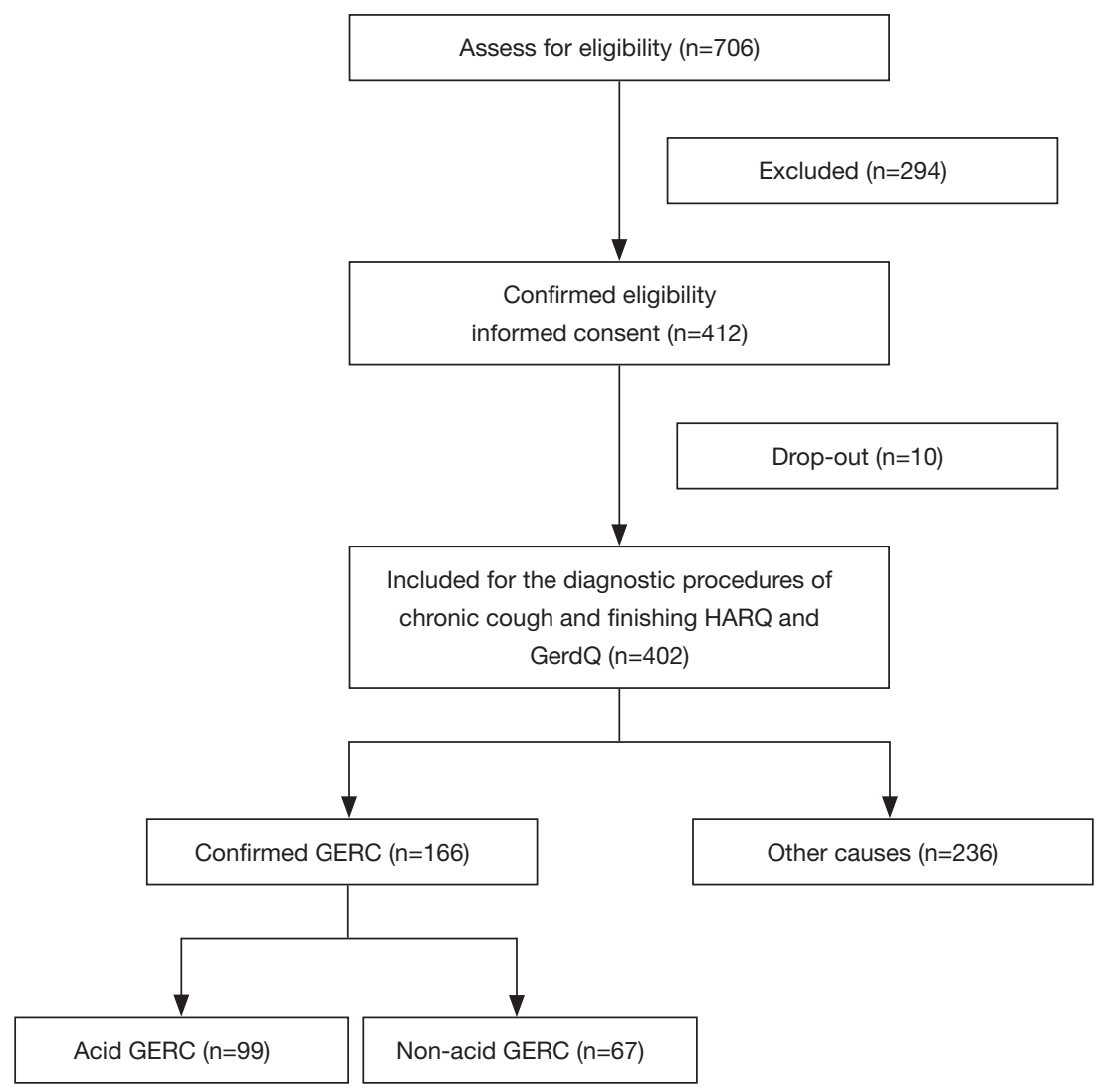

Figure 1 The flow of patients through the study. HARQ, Hull airway reflux questionnaire; GerdQ, gastroesophageal reflux disease questionnaire; GERC, gastroesophageal reflux-induced chronic cough.

daily) for 4 weeks. If a favorable response occurred, the treatment was maintained until cough resolution was achieved.

\section{Study design}

This was a prospective clinical trial. After the medical history review and physical examination, chest X-ray/CT scan, lung function examination, bronchial provocation test, an assay of cells in induced sputum and MII-pH were completed according to the diagnostic procedures of chronic cough, aiming to identify a cause of chronic cough (8). All diagnostic and treatment processes followed the 2015 Guideline for the Diagnosis and Treatment of Cough (8). Meanwhile, HARQ and GerdQ were also conducted by the first author. Once upper airway cough syndrome (UACS), cough variant asthma (CVA), eosinophilic bronchitis (EB), GERC or atopic cough (AC) was suspected, treatment targeting the cause of cough was initiated, aiming to confirm the diagnosis. When all the examination mentioned above showed negative, empirical therapy targeting the cause in the order of UACS, CVA, $\mathrm{EB}, \mathrm{AC}$, and GERC was performed. The diagnosis was confirmed or excluded according to the response to a specific therapy, and the potential cause was explored. During the therapy, patients were followed up by clinic visit once every 2 weeks. Figure 1 shows the flow of patients through the study.

\section{Laboratory investigation}

\section{Cough symptom score}

The cough symptom score reported by Hsu et al. (11) was used (Table 1). Cough symptom score: Cough symptom score was performed with the scale developed by Hsu et al. (11). This scale was divided into two parts (daytime and nighttime cough symptom scores), and the score ranged from 0 to 5 , with 0 being best (no cough) and 5 being worst 
Table 1 Cough symptom score

\begin{tabular}{ll}
\hline Daytime & Nighttime \\
\hline $0=$ no cough during the day & $0=$ no cough during the night \\
$1=$ cough for one short period & $1=$ cough on waking only \\
$2=$ cough for more than two short periods & $2=$ wake once or early due to cough \\
$3=$ frequent coughing, which did not interfere with usual daytime & $3=$ frequent waking due to coughs activities \\
$4=$ frequent coughing, which did interfere with usual daytime activities & $4=$ frequent coughs most of the night \\
$5=$ distressing coughs most of the day & $5=$ distressing coughs preventing any sleep \\
\hline
\end{tabular}

Table 2 Hull airway reflux questionnaire (HARQ)

\begin{tabular}{lllll}
\hline & & \multicolumn{3}{c}{ Point } \\
\cline { 2 - 4 } Within the last month, how did the following problems affect you? & 0 & 1 & 2 & 3 \\
\hline
\end{tabular}

Hoarseness or a problem with your voice

Clearing your throat

The feeling of something dripping down the back of your nose or throat

Retching or vomiting when you cough

Cough on first lying down or bending over

Chest tightness or wheeze when coughing

Heartburn, indigestion, stomach acid coming up (or do you take medications

for this, if yes score 5)

A tickle in your throat, or a lump in your throat

Cough with eating (during or soon after meals)

Cough with certain foods

Cough when you get out of bed in the morning

Cough brought on by singing or speaking (for example, on the telephone)

Coughing more when awake rather than asleep

A strange taste in your mouth

$0=$ no problem and $5=$ severe/frequent problem.

(characterized by a distressing cough).

\section{HARQ}

HARQ is a patient self-assessment questionnaire (Table 2). It consists of 14 question items, each of which refers to cough trigger or exacerbation factors, or the severity of concomitant symptoms such as postnasal drip, acid reflux or heartburn. The questionnaire asks the patients to recall the frequency of each symptom within last month, which was divided into 6 levels according to the degree. The answer is a 6 -point scoring method from 0 to 5 . The score increases with the frequency of symptoms. In the past month, it was considered as 0 point when the symptom did not appear, and it was considered as 5 points when the symptom was severe or frequently appeared. The total score ranges from 0 to 70 points. The Chinese version of HARQ (7) was used with the consent and authorization of HARQ designer Morice. All patients and investigators were blinded to HARQ scores throughout treatments during the study.

\section{GerdQ}

GerdQ (Table 3) consists of six problems of symptom, 
Table 3 Gastroesophageal reflux disease questionnaire (GerdQ)

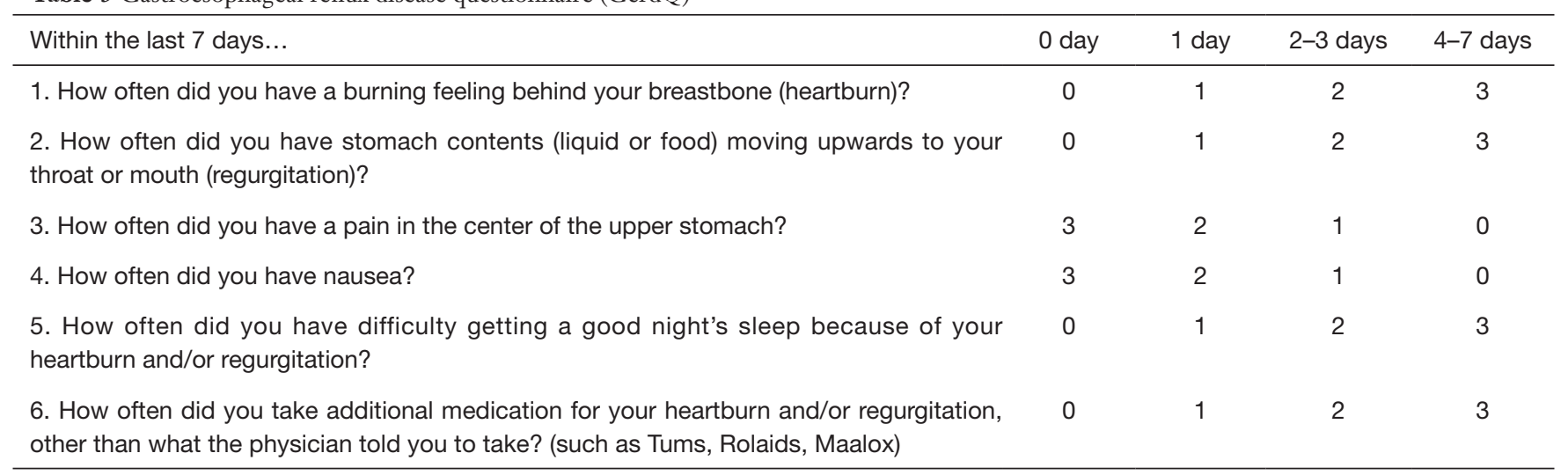

Questions 1, 2, 5 and 6 are reflux positively related; questions 3 and 4 are reflux negatively related.

including four reflux positively related questions and two reflux negatively related questions (12). The questionnaire asks patients to recall the frequency of each symptom in the past week, which was divided into 4 levels ( $0-3$ points) according to the degree. The total score range was from 0 to 18 points. Higher scores indicated higher possibilities of gastroesophageal reflux disease. All patients and investigators were blinded to GerdQ scores throughout treatments during the study.

\section{MII-pH}

MII-pH was performed as previously described (13) after the patients had stopped taking acid suppressing medication for at least 1 week. Briefly, a combined MII-pH catheter was transnasally inserted into a patient's esophagus, with six impedance channel sensors (K6011-E10632, Unisensor, Switzerland) located 3, 5, 7, 9, 15 and $17 \mathrm{~cm}$ above the lower esophageal sphincter, and an antimony $\mathrm{pH}$ electrode (819100, Medical Measurement System B.V., Netherlands) positioned $5 \mathrm{~cm}$ above the proximal border of the lower esophageal sphincter. A connected portable data logger (Ohmega, Medical Measurement System B.V., Netherlands) stored data from all seven channels over $24 \mathrm{~h}$. Reflux episodes recorded on the tracings of MII-pH were manually characterized by their impedance value as liquid, gas or mixed liquid-gas reflux or characterized by $\mathrm{pH}$-metry as acid $(\mathrm{pH}<4.0)$, weakly acidic $(4.0<\mathrm{pH}<7.0)$ or weakly alkaline $(\mathrm{pH}>7.0)$ reflux, with the latter two collectively referred to as non-acid reflux. The DeMeester score was calculated as a global measure of esophageal acid exposure. SAP was used to represent the temporal association between cough recorded by patients on diary cards and reflux which had occurred during the preceding 2 -min period $(13,14)$.

\section{Induced sputum cytology}

Induced sputum was performed according to previously described protocol (1). Finally, differential cell count was conducted manually by counting 400 nucleated cells.

\section{Bronchial provocation test}

Pulmonary function and histamine bronchial provocation tests were performed according to the methods recommended by the Respiratory Branch of the Chinese Medical Association. The instruments adopted were MasterScreen Diffusion lung function instrument and APS nebulizer from Jaeger Company (Germany). With histamine as the stimulant, when the cumulative histamine dose $\left(\mathrm{PD} 20-\mathrm{FEV}_{1}\right)$ that reduces $\mathrm{FEV}_{1}$ by $20 \%$ was less than $7.8 \mathrm{~mol}$, the increased airway reactivity was considered. All indeterminate results of the above tests were conducted for a second time, and conclusive results were finally got. No significant adverse events occurred during above tests.

\section{Statistical analysis}

Statistical analysis was performed by using SPSS 24.0 statistical software package (IBM, USA). Normally distributed data are expressed as mean \pm standard deviation (SD), while those with skewed distribution were expressed as medians (25-75\% interquartile). Categorical variables are presented in percentage (\%) and numbers. The $t$-test was used to compare data between the two groups when variances were uniform, and the Mann-Whitney $\mathrm{U}$ test was used to compare data between the two groups when variances were uneven. Multiple groups were compared using the ANOVA method. In order to avoid the interaction between multiple etiologies, this study only performed 
Table 4 Basic information for all enrolled chronic cough patients

\begin{tabular}{ll}
\hline Items & Mean \pm SD or ratio \\
\hline Age $(\mathrm{yrs})$ & $46.58 \pm 15.68$ \\
Gender $(\mathrm{F} / \mathrm{M})$ & $152 / 250$ \\
Course of cough $(\mathrm{m})$ & $12.00(32.00)$ \\
HARQ & $22.38 \pm 8.54$ \\
GerdQ & $7.18 \pm 1.60$ \\
Cough symptom score & \\
Daytime & $2.99 \pm 0.82$ \\
Nighttime & $1.70 \pm 1.41$ \\
Lung function $(\%, \bar{x} \pm \mathrm{S})$ & \\
FEV ${ }_{1}$ predicted $(\%)$ & $98.96 \pm 14.28$ \\
FVC predicted $(\%)$ & $106.28 \pm 63.89$ \\
FEV $/$ FVC\% & $81.56 \pm 8.97$ \\
\hline
\end{tabular}

$\mathrm{SD}$, standard deviation; $\mathrm{HARQ}$, Hull airway reflux questionnaire; GerdQ, gastroesophageal reflux disease questionnaire; FEV forced expiratory volume in 1 second; FVC, forced vital capacity.

ROC and logistic regression analysis on patients with the single etiology. ROC curve was used to evaluate the diagnostic efficacy of HARQ score and GerdQ score in acid GERC and non-acid GERC respectively. The Logistic regression model was used to fit the HARQ and GerdQ scores to generate a joint predictor $(15,16)$. ROC curve was used to evaluate the diagnostic efficacy of HARQ score, GerdQ score, and HARQ score in combination of GerdQ score in GERC, and the area under the curve, sensitivity (\%), specificity (\%), positive predictive value (\%), negative predictive value (\%), Youden index were calculated. The best predicted diagnostic value was given. The statistical significance of the improvement in AUC was calculated by DeLong's test (17). $\mathrm{P}<0.05$ was considered statistically significant.

\section{Results}

\section{General clinical information}

During the study, 706 consecutive patients with chronic cough were treated in our outpatient department from January 2015 to July 2019. A total of 412 patients met the inclusion criteria. Four people dropped out from the study because of incomplete data eventually. Six people were lost to the follow-up. Five GERC patients diagnosed through empirical anti-reflux treatment were excluded because of inability to distinguish between acid and non-acid GERC. Thus, 402 (402/412, 97.6\%) patients with chronic cough were finally included, including 152 male and 250 female patients, with an average age of $46.58 \pm 15.68$ years old (Table 4).

Of the 402 patients with chronic cough, 248 (61.69\%) underwent bronchial provocation tests and 254 (63.18\%) underwent induced sputum testing. Among the 402 patients with chronic cough, there were $332(82.59 \%)$ patients with the single etiology, $56(13.93 \%)$ patients with dual etiologies, 4 (0.99\%) patients with three etiologies, and 10 (2.49\%) patients with unexplained etiologies. Of the 332 patients with the single etiology, 114 (28.36\%) were GERC patients, $72(17.91 \%)$ were CVA patients, $53(13.18 \%)$ were AC patients, 43 (10.70\%) were EB patients, and 26 (6.47\%) were UACS patients (Table 5). There were totally 166 GERC patients diagnosed by MII-pH, of which 99 (59.6\%) were diagnosed with acid GERC and 67 (40.4\%) were diagnosed with non-acid GERC.

\section{Comparison of HARQ and GerdQ in patients with different etiologies}

Among the 402 patients with chronic cough, the HARQ scores of GERC patients was significantly higher than that of non-GERC patients $(27.60 \pm 7.54$ vs. $18.71 \pm 7.20$, $\mathrm{t}=-11.860, \mathrm{P}<0.001)$. Among 166 GERC patients, there was no significant difference in HARQ score between acid GERC and non-acid GERC patients $(28.32 \pm 7.51$ vs. $26.57 \pm 7.50, t=1.461, P=0.146)$. The differences in HARQ scores among GERC, EB, AC, UACS, and CVA patients were statistically significant $(\mathrm{F}=23.188, \mathrm{P}<0.001)$, and only the HARQ score in GERC patients $(27.57 \pm 7.92)$ was significantly higher than those in the EB $(19.95 \pm 7.87$, $\mathrm{t}=5.402)$, AC $(19.49 \pm 6.98, \mathrm{t}=6.667)$, UACS $(18.58 \pm 7.10$, $\mathrm{t}=5.704)$ and CVA $(18.22 \pm 7.59, \mathrm{t}=8.048)$ patients (all $\mathrm{P}<0.001$ ) (Figure 2).

The GerdQ score of GERC patients was significantly higher than that of non-GERC patients $(8.48 \pm 1.66 \mathrm{vs}$. $6.26 \pm 0.66, \mathrm{t}=-18.513, \mathrm{P}<0.001)$. Among the 166 GERC patients, the GerdQ score of non-acid GERC patients was significantly lower than that of acid GERC patients (7.28 \pm 1.31 vs. $8.75 \pm 1.56, \mathrm{t}=6.528, \mathrm{P}<0.001)$. The differences in GerdQ scores among GERC, EB, AC, UACS, and CVA patients were statistically significant $(\mathrm{F}=61.104, \mathrm{P}<0.001)$. Only the GerdQ score in GERC patients $(8.23 \pm 1.54)$ was significantly higher than those in the CVA $(6.36 \pm 0.59$, 
Table 5 Etiologies of chronic cough patients

\begin{tabular}{|c|c|}
\hline Etiology & Cases, n (\%) \\
\hline Single etiology & $332(82.59)$ \\
\hline GERC & $114(28.36)$ \\
\hline CVA & $72(17.91)$ \\
\hline$A C$ & $53(13.18)$ \\
\hline EB & $43(10.70)$ \\
\hline UACS & $26(6.47)$ \\
\hline Post infectious cough & $12(2.96)$ \\
\hline ACEI-related chronic cough & $8(1.99)$ \\
\hline Psychogenic cough & $3(0.75)$ \\
\hline Premature beat induced cough & $1(0.25)$ \\
\hline Dual etiologies & $56(13.93)$ \\
\hline GERC + AC & $20(4.98)$ \\
\hline GERC + EB & $19(4.73)$ \\
\hline GERC + CVA & $7(1.74)$ \\
\hline GERC + UACS & $2(0.50)$ \\
\hline $\mathrm{EB}+\mathrm{UACS}$ & $6(1.49)$ \\
\hline ACEI-related cough + CVA & $1(0.25)$ \\
\hline ACEl-related cough $+\mathrm{AC}$ & $1(0.25)$ \\
\hline Three etiologies & $4(0.99)$ \\
\hline GERC + AC + UACS & $2(0.50)$ \\
\hline GERC + EB + UACS & $1(0.25)$ \\
\hline GERC + CVA + UACS & $1(0.25)$ \\
\hline Unexplained etiologies & $10(2.49)$ \\
\hline Total & 402 \\
\hline
\end{tabular}

GERC, gastroesophageal reflux-induced chronic cough; CVA, cough variant asthma; $A C$, atopic cough; EB, eosinophilic bronchitis; UACS, upper airway cough syndrome; ACEI, angiotensin-converting enzyme inhibitor.

$\mathrm{t}=9.834), \mathrm{EB}(6.30 \pm 0.94, \mathrm{t}=7.669), \mathrm{AC}(6.15 \pm 0.46, \mathrm{t}=9.610)$, UACS $(6.15 \pm 0.46, \mathrm{t}=6.780)$ patients (all $\mathrm{P}<0.001)$ (Figure 3).

\section{Comparison of MII-pH between acid GERC and non-acid GERC}

DeMeester scores, acid SAP, acid reflux, and acid clearance time were significantly higher in patients with acid GERC than in patients with non-acid GERC $(\mathrm{P}<0.01)$, and nonacid SAP in patients with non-acid GERC was significantly higher than patients with acid GERC ( $\mathrm{P}=0.001)$. However, the number of weak acid reflux, weak base reflux, gas reflux, liquid reflux, mixed reflux was not statistically different between patients with acid GERC and non-acid GERC $(\mathrm{P}>0.05)$ (Table 6).

\section{Predictive diagnostic value of $H A R Q$ and GerdQ score alone for acid and non-acid GERC}

When HARQ and GerdQ was used in the predictive diagnosis of acid GERC, the AUC of GerdQ ROC (0.928) was significantly higher than that of HARQ (0.797) (DeLong's test, $\mathrm{P}=0.0001$ ) (Figure $4 A$ and Table 7). When HARQ and GerdQ was used in the predictive diagnosis of non-acid GERC, the AUC of HARQ ROC (0.683) was significantly higher than that of GerdQ (0.524) (DeLong's test, $\mathrm{P}=0.0003$ ) (Figure $4 B$ and Table 7).

\section{The predictive diagnostic value of $H A R Q$, GerdQ score alone and their combination for GERC}

When HARQ was used in the predictive diagnosis of GERC, the AUC was 0.796, and the sensitivity and specificity was $77.19 \%$ and $77.06 \%$, respectively. When GerdQ was used in the predictive diagnosis of GERC, the AUC was 0.763 , and the sensitivity and specificity was $70.18 \%$ and $76.15 \%$, respectively. When GerdQ was combined with HARQ in the predictive diagnosis of GERC, a binary logistic regression analysis is performed. The linear model is $\operatorname{Logit}(\mathrm{P})=-8.530+0.127 \times$ HARQ $+0.631 \times$ GerdQ. When it was used in the predictive diagnosis of GERC, the AUC was improved to 0.848 (DeLong's test, $\mathrm{P}=0.0006$ ), and the sensitivity and specificity was $77.19 \%$ and $79.82 \%$, respectively (Figure $4 C$ and Table 8). Table 9 shows the cross tabulation of the HARQ and GerdQ in relation to the diagnosis of GERC.

\section{Discussion}

GERC is one of the common causes of chronic cough (2). In the past, the proportion of GERC in chronic cough in European and American countries was slightly higher than that in Asian countries (18-22). However, in the recent 20 years, with the increase in the incidence of gastroesophageal reflux disease, the incidence of GERC has increased year by year (23-25). Results of the epidemiological survey in our department in the past 8 years show that the incidence of GERC in chronic cough 

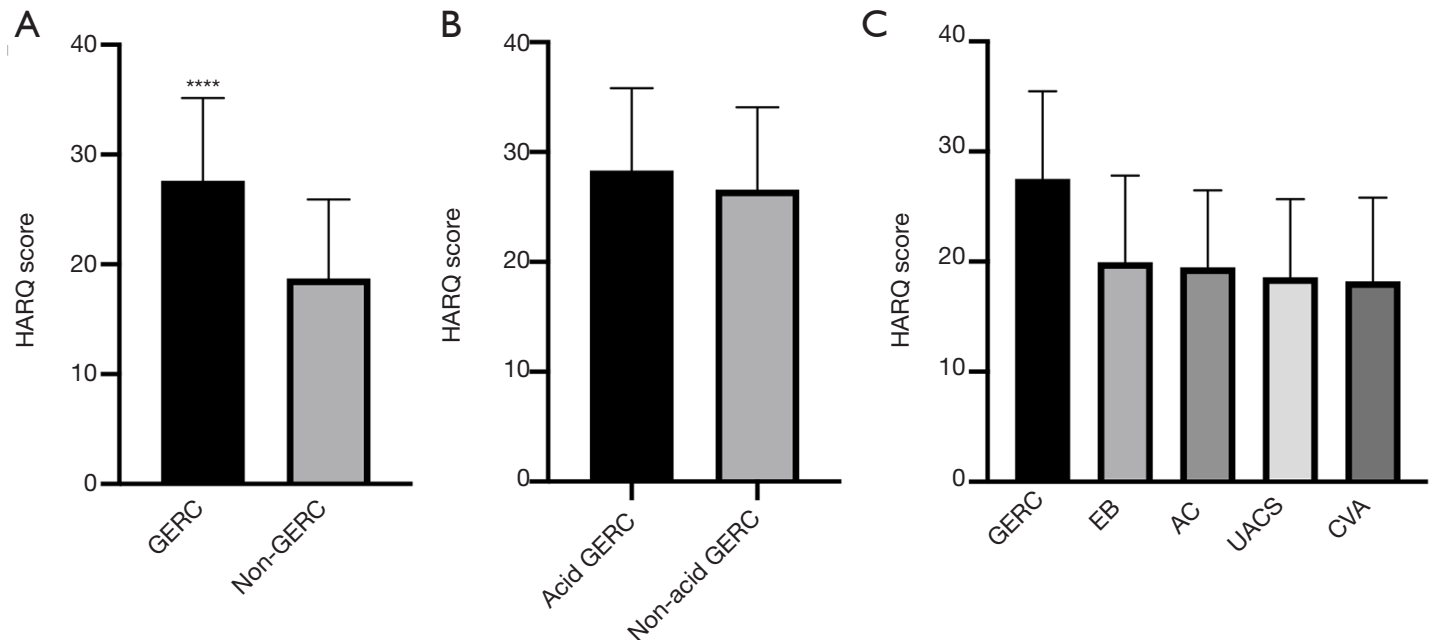

Figure 2 Comparison of the HARQ score between different groups. (A) Comparison of the HARQ score between GERC and non-GERC patients; (B) comparison of the HARQ score between acid GERC and non-acid GERC patients; (C) comparison of the HARQ score among GERC, EB, AC, UACS and CVA patients. ***, P<0.0001. HARQ, Hull airway reflux questionnaire; GERC, gastroesophageal refluxinduced chronic cough; EB, eosinophilic bronchitis; AC, atopic cough; UACS, upper airway cough syndrome; CVA, cough variant asthma.
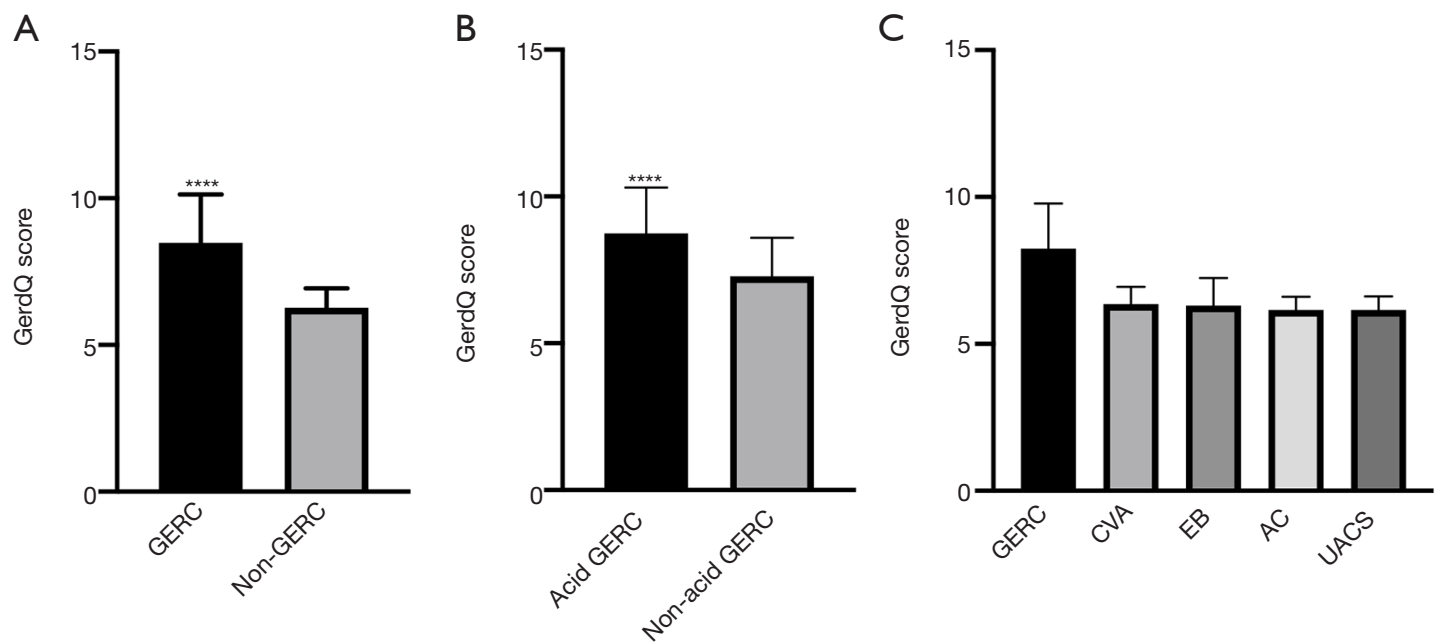

Figure 3 Comparison of the GerdQ score between different groups. (A) Comparison of the GerdQ score between GERC and non-GERC patients; (B) comparison of the GerdQ score between acid GERC and non-acid GERC patients; (C) comparison of the GerdQ score among GERC, EB, AC, UACS and CVA patients. ${ }^{* * * *}, \mathrm{P}<0.0001$. GerdQ, gastroesophageal reflux disease questionnaire; GERC, gastroesophageal reflux-induced chronic cough; EB, eosinophilic bronchitis; AC, atopic cough; UACS, upper airway cough syndrome; CVA, cough variant asthma.

has increased from $15.4 \%$ to $40.7 \%$ (1). The increase in the incidence of GERC may be attributed to the increased gastric acid secretion (26), the doctors' increased awareness of gastroesophageal reflux disease, and the continuous improvement of diagnostic methods (27).

As the morbidity of GERC increases, the diagnosis and treatment of GERC becomes more and more important. At present, the detection method of GERC has been greatly improved, which is also one of the reasons for the increased morbidity. However, there are still certain limitations $(24,27,28)$. Barium meal of the upper gastrointestinal tract can show barium reflux, and gastroscopy can visually show 
Table 6 Comparison of different variables of MII-pH between acid GERC and non-acid GERC

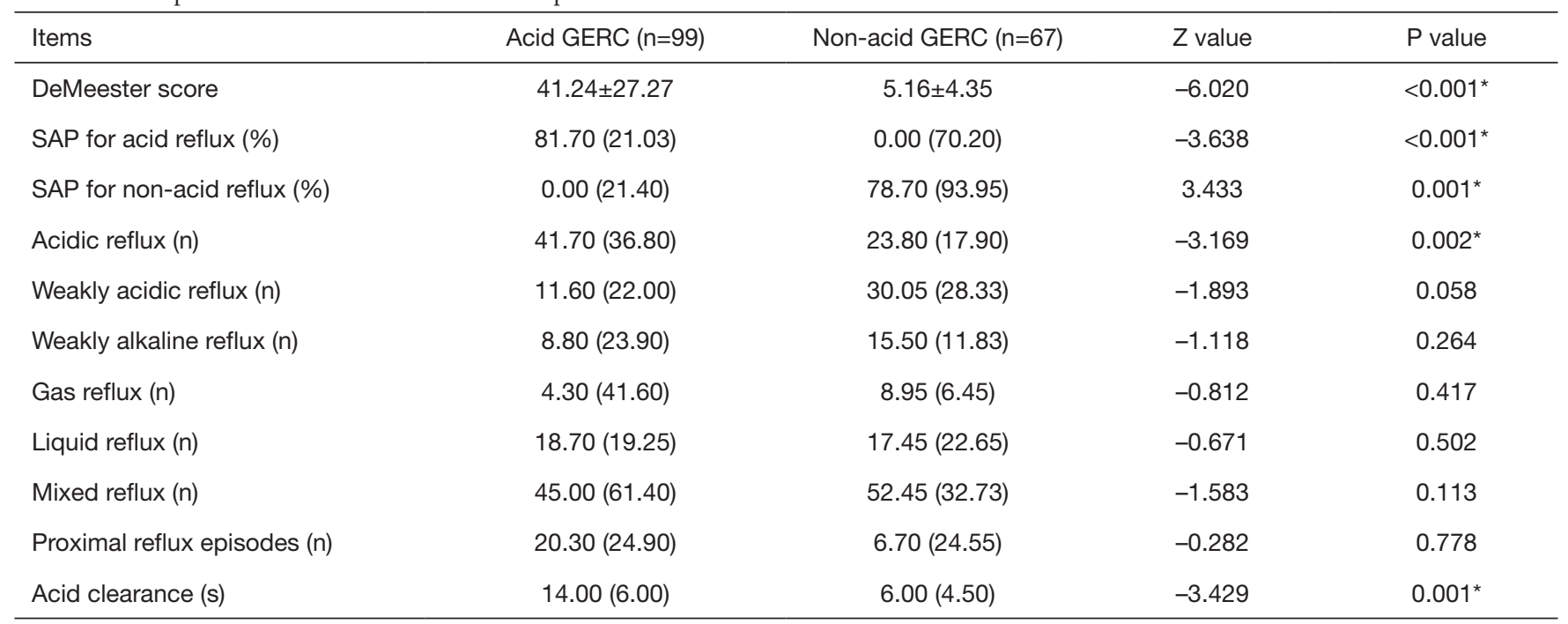

*, $\mathrm{P}<0.01$ denotes the statistical significance. $\mathrm{MII}-\mathrm{pH}$, multichannel intraluminal esophageal impedance and $\mathrm{pH}$ monitoring; GERC, gastroesophageal reflux-induced chronic cough; SAP, syndrome association probability.
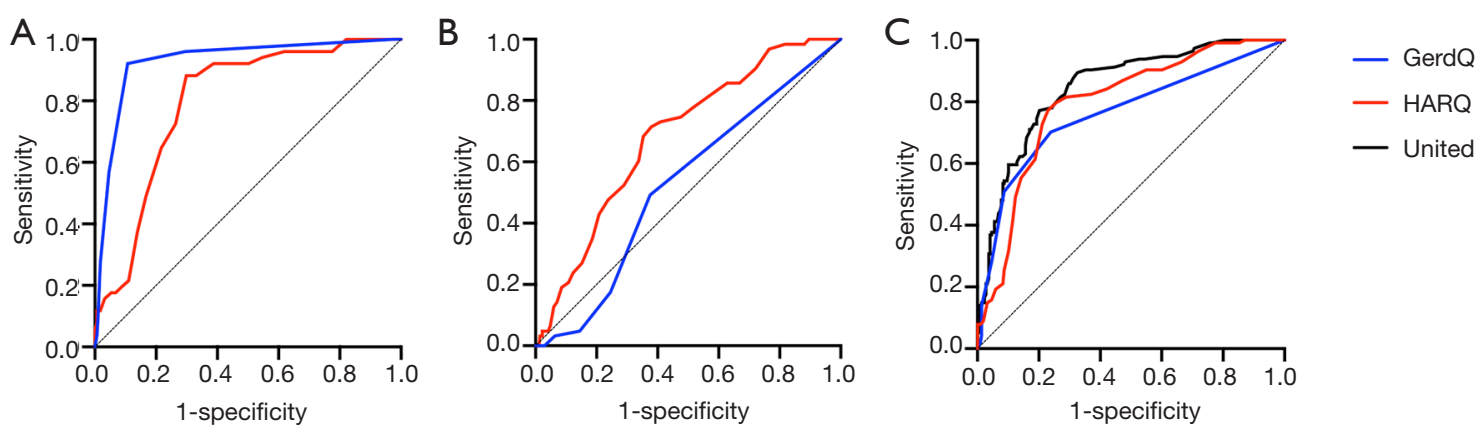

Figure 4 Diagnostic value of HARQ and GerdQ in GERC. (A) Receiver operating cure of HARQ and GerdQ in predicting acid GERC; (B) receiver operating cure of HARQ and GerdQ in predicting non-acid GERC; (C) receiver operating cure of HARQ, GerdQ, and the combination of HARQ and GerdQ in predicting GERC. Blue line: GerdQ; red line: HARQ; black line: the combination of HARQ and GerdQ. HARQ, Hull airway reflux questionnaire; GerdQ, gastroesophageal reflux disease questionnaire; GERC, gastroesophageal refluxinduced chronic cough.

inflammation of the esophageal mucosa, while the false negative rates of these two methods are very high (29), making it easy to miss the diagnosis of GERC without visible damages to the esophageal mucosa. MII-pH is currently the most sensitive and specific test for diagnosing GERC (30), which can accurately detect abnormal acid and non-acid refluxes with or without esophageal mucosal damages, and find the correlation between the reflux and cough, improving the diagnostic efficiency of GERC. However, MII-pH is an invasive examination. Some patients cannot tolerate it. Thus, it has not yet been universal in clinics. Besides, it is also relatively expensive, and the diagnostic standards have not yet reached consensus in different countries. The determination of SAP depends on the detailed cough time recorded by patients, while some patients cannot co-ordination perfectly, leading to serious deviations in data. Therefore, empirical anti-reflux therapy is still an important method for the diagnosis and treatment of GERC. However, some GERC patients may not have typical symptoms such as acid refluxes and heartburn. Our previous research indicates that the proportions of acid refluxed and heartburn in patients with acid GERC are only $57 \%$ and $33 \%$ respectively, though they are significantly higher than those in non-acid GERC patients (31). The 
Table 7 Prediction of acid and non-acid GERC with HARQ or GerdQ

\begin{tabular}{|c|c|c|c|c|}
\hline Items & \multicolumn{2}{|c|}{ Acid-GERC } & \multicolumn{2}{|c|}{ Non-acid GERC } \\
\hline $\mathrm{ROC}$ & 0.797 & 0.928 & 0.683 & 0.524 \\
\hline Cut-off value & 24.5 & 8.5 & 22.5 & 7.5 \\
\hline Youden index & 0.583 & 0.815 & 0.335 & 0.117 \\
\hline Specificity (\%) (95\% Cl) & $70.11(64.33,75.33)$ & $89.32(84.97,92.57)$ & $62.08(55.96,67.85)$ & $62.45(56.34,68.20)$ \\
\hline $\begin{array}{l}\text { Positive predictive value (\%) } \\
(95 \% \mathrm{Cl})\end{array}$ & $34.89(26.85,43.83)$ & $61.03(49.22,71.74)$ & $30.61(23.43,38.83)$ & $23.48(16.74,31.81)$ \\
\hline $\begin{array}{l}\text { Negative predictive value (\%) } \\
(95 \% \mathrm{Cl})\end{array}$ & $97.05(93.38,98.79)$ & $98.43(95.76,99.50)$ & $90.27(84.83,93.97)$ & $84.00(78.01,88.65)$ \\
\hline
\end{tabular}

GERC, gastroesophageal reflux-induced chronic cough; HARQ, Hull airway reflux questionnaire; GerdQ, gastroesophageal reflux disease questionnaire.

Table 8 Prediction of GERC with HARQ or GerdQ

\begin{tabular}{llll}
\hline Items & HARQ & GerdQ & HARQ \& GerdQ \\
\hline ROC & 0.796 & 0.763 & 0.848 \\
Cut-off value & 23.5 & 7.5 & -0.7475 \\
Youden index & 0.543 & 0.763 & 0.571 \\
Sensitivity $(\%)(95 \% \mathrm{Cl})$ & $77.19(68.21,84.31)$ & $70.18(60.77,78.19)$ & $77.19(68.21,84.31)$ \\
Specificity $(\%)(95 \% \mathrm{Cl})$ & $77.06(70.80,82.35)$ & $76.15(69.82,81.53)$ & $79.82(73.75,84.81)$ \\
Positive predictive value $(\%)(95 \% \mathrm{Cl})$ & $63.76(55.11,71.65)$ & $60.61(51.70,68.89)$ & $66.67(57.86,74.48)$ \\
Negative predictive value $(\%)(95 \% \mathrm{Cl})$ & $86.60(80.80,90.90)$ & $83.00(76.91,87.79)$ & $87.00(81.35,91.18)$ \\
$\chi^{2}$ value & 90.726 & 67.062 & 101.576 \\
P value in $\chi^{2}$ test & $<0.001$ & $<0.001$ & $<0.001$ \\
$\kappa$ value & 0.517 & 0.446 & 0.549 \\
P value in $\kappa$ test & $<0.001$ & $<0.001$ & $<0.001$ \\
\hline
\end{tabular}

HARQ \& GerdQ: $-8.530+0.127 \times \mathrm{HARQ}+0.631 \times$ GerdQ. GERC, gastroesophageal reflux-induced chronic cough; HARQ, Hull airway reflux questionnaire; GerdQ, gastroesophageal reflux disease questionnaire.

recent ERS guideline does not recommend the proton pump inhibitor treatment for such patients either (32). Therefore, the empirical anti-reflux therapy has defects such as poor targeting and low treatment efficiency. What's more, some patients who fail to be cured with the empirical anti-reflux therapy cannot be completely ruled out the possibility of GERC due to the possible poor compliance of patients, the inadequate dosage and the inappropriate therapy course. Therefore, we are committed to finding a simple, non-invasive and handy evaluation method for

\section{GERC.}

The results of this study show that the GerdQ score of GERC patients are significantly higher than that of chronic cough patients with other etiologies, and ROC analysis results show that the GerdQ score has a limited diagnostic value for GERC which is consistent with the previous research (5). According to the different properties of refluxes, GERC can be divided into acid GERC and non-acid GERC (31). The reflux with a $\mathrm{pH}<4$ is defined as the acid reflux, $\mathrm{pH}$ between 4 and 7 is the weak acid reflux, 
Table 9 Cross tabulation of the HARQ and GerdQ in relation to diagnosis of GERC

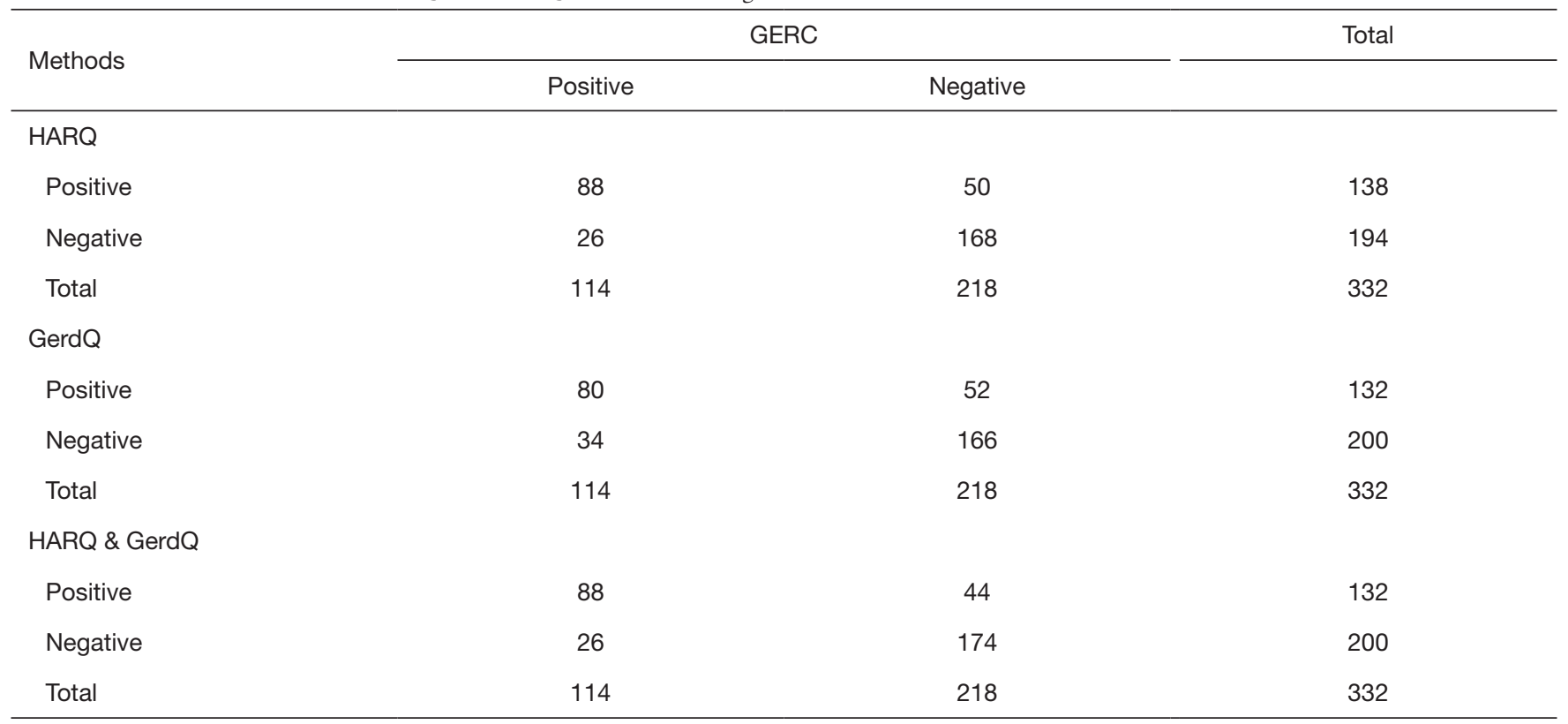

HARQ \& GerdQ: $-8.530+0.127 \times \mathrm{HARQ}+0.631 \times$ GerdQ. HARQ, Hull airway reflux questionnaire; GerdQ, gastroesophageal reflux disease questionnaire; GERC, gastroesophageal reflux-induced chronic cough.

and $\mathrm{pH}>7$ is the weak alkali reflux (33). The incidence of acid refluxes and belching in non-acid GERC patients is significantly lower than that in acid GERC patients $(34,35)$, so the GerdQ score in patients with non-acid GERC was significantly lower than that in acid GERC patients. The GerdQ score only has advantages in distinguishing acid and non-acid GERC (5), suggesting that the GerdQ score is helpful for the diagnosis of acid GERC, while the diagnostic value for non-acid GERC is not high. The results of this study show that the GerdQ score have a low sensitivity and specificity in the predictive diagnosis of non-acid GERC, similar to the results of our previous research (31). The GerdQ score of non-acid GERC patients is not high, indicating that the GerdQ score lacks the diagnostic value for non-acid GERC, which may eventually lead to the missed diagnosis of some non-acid GERC patients due to the low GerdQ score. The reason may be that most of the contents of GerdQ is related to typical symptoms such as acid reflux and heartburn, and gastric acid and pepsin that cause these symptoms due to damages of the esophageal mucosa mainly exist in acid refluxes. Thus, the GerdQ score can only effectively reflect the acid reflux caused symptoms. Besides, the GerdQ score lacks coughrelated questions, which could underestimate the chronic cough.
HARQ is a simple assessment tool designed by Morice for the cough hypersensitivity syndrome $(2,3,6)$. Multinational clinical studies have confirmed its high sensitivity and specificity for the assessment of the airway reflux and cough hypersensitivity $(3,7,36)$. Unlike other cough questionnaires, Morice designed HARQ to find the cause of chronic cough, rather than as a simple assessment (2). Compared with GerdQ, HARQ adds the evaluation of chronic cough. Meanwhile, the 14 items include the evaluation of four gastroesophageal refluxrelated symptoms such as cough, heartburn, and acid reflux at lying down or bending down. Thus, HARQ has an assessment of both chronic cough and gastroesophageal reflux symptoms. Similar to our previous study (7), the HARQ value of GERC patients in this study is significantly higher than that of non-GERC patients. Unlike GerdQ, HARQ has a similar predictive diagnostic value of acid and non-acid GERC, which may be because HARQ does not focus purely on the typical acid reflux symptoms such as refluxes, heartburn, and acid refluxes.

To further improve the predictive diagnostic value of HARQ for GERC, make up for the diagnostic defects of GerdQ for non-acid GERC, improve the diagnosis efficiency, and avoid missed diagnosis, HARQ in combination with GerdQ was firstly used in the predictive 
diagnosis of GERC. A linear model including HARQ and GerdQ was established through Logistic analysis, showing that the combination has a significantly higher predictive diagnostic value for GERC, and the sensitivity, specificity, positive predictive value, and negative predictive value were higher than HARQ or GerdQ alone in the predictive diagnosis of GERC. Therefore, when combined with GerdQ, HARQ can not only help to make up for the defects of GerdQ in the diagnosis of non-acid GERC, but also increase the assessment of chronic cough, thus increasing the predictive diagnostic value of GERC. This linear model can provide the diagnostic and therapeutic basis for patients with suspected GERC who cannot tolerate invasive tests such as MII-pH, and the diagnosis efficiency is higher than HARQ or GerdQ alone. However, this model only includes the HARQ and GerdQ scores, which cannot completely replace MII-pH currently.

There are still some limitations in this study. According to the latest Lyon Consensus (37) of 2019, acid exposure time and reflux events are added to the diagnostic criteria of gastroesophageal reflux disease. However, there is no such standard at the beginning of this study. Nevertheless, results of this study show that acid GERC patients have significantly higher acid clearance times than non-acid GERC patients, and acid reflux time is significantly higher than that in non-acid GERC patients, which is consistent with the diagnostic criteria of Lyon Consensus. More studies are needed using the diagnostic criteria of Lyon Consensus in the future.

In conclusion, HARQ used to evaluate cough hypersensitivity has a certain predictive diagnostic value for GERC. The diagnosis of GERC should be considered when the HARQ score is $\geq 24$ points. The predictive diagnostic value of HARQ and GerdQ combination is higher than HARQ or GerdQ alone. The combination can help to diagnose GERC simply, quickly and effectively in clinic.

\section{Acknowledgments}

Funding: This study was supported by the National Natural Science Foundation of China (No. 82070102 and 81770097), the Project of Science and Technology Commission of Shanghai Municipality (No. 20ZR1451500 and 17411970800), the Fund of Shanghai Youth Talent Support Program, the Fund of Shanghai Municipal Health Commission for Excellent Young Scholars (No. 2018YQ01) and the Shanghai Sailing Program (No. 19YF1444100).

\section{Footnote}

Reporting Checklist: The authors have completed the STARD reporting checklist. Available at http://dx.doi.org/10.21037/ atm-20-3236

Data Sharing Statement: Available at http://dx.doi. org/10.21037/atm-20-3236

Peer Review File: Available at http://dx.doi.org/10.21037/ atm-20-3236

Conflicts of Interest: All authors have completed the ICMJE uniform disclosure form (available at http://dx.doi. org/10.21037/atm-20-3236). The authors have no conflicts of interest to declare.

Ethical Statement: The authors are accountable for all aspects of the work in ensuring that questions related to the accuracy or integrity of any part of the work are appropriately investigated and resolved. The study was conducted in accordance with the Declaration of Helsinki (as revised in 2013). The study was approved by the Ethics Committee of our Hospital (No. K-2015-007) and registered in the Chinese Clinical Trials Register (http:// www.chictr.org.cn/) (ChiCTR-DDD-17012869). Informed consent was taken from all the patients.

Open Access Statement: This is an Open Access article distributed in accordance with the Creative Commons Attribution-NonCommercial-NoDerivs 4.0 International License (CC BY-NC-ND 4.0), which permits the noncommercial replication and distribution of the article with the strict proviso that no changes or edits are made and the original work is properly cited (including links to both the formal publication through the relevant DOI and the license). See: https://creativecommons.org/licenses/by-nc-nd/4.0/.

\section{References}

1. Ding $\mathrm{H}, \mathrm{Xu} \mathrm{X}$, Wen $\mathrm{S}$, et al. Changing etiological frequency of chronic cough in a tertiary hospital in Shanghai, China. J Thorac Dis 2019;11:3482-9.

2. Irwin RS. Chronic cough due to gastroesophageal reflux disease: ACCP evidence-based clinical practice guidelines. Chest 2006;129:80S-94S.

3. Morice AH, Fontana GA, Sovijarvi AR, et al. The 
diagnosis and management of chronic cough. Eur Respir J 2004;24:481-92.

4. Kanemitsu Y, Kurokawa R, Takeda N, et al. Clinical impact of gastroesophageal reflux disease in patients with subacute/chronic cough. Allergol Int 2019;68:478-85.

5. Xu X, Chen Q, Liang S, et al. Comparison of gastroesophageal reflux disease questionnaire and multichannel intraluminal impedance $\mathrm{pH}$ monitoring in identifying patients with chronic cough responsive to antireflux therapy. Chest 2014;145:1264-70.

6. Morice AH, Faruqi S, Wright CE, et al. Cough hypersensitivity syndrome: a distinct clinical entity. Lung 2011;189:73-9.

7. Huang Y, Yu L, Xu XH, et al. Validation of the Chinese version of Hull airway reflux questionnaire and its application in the evaluation of chronic cough. Zhonghua Jie He He Hu Xi Za Zhi 2016;39:355-61.

8. Chinese Medical Association of Respiratory Diseases Association Asthma Group. Diagnosis and Treatment Guideline of Cough (2015). Chin J Tubercul Res 2016;39:323-54.

9. Dong $\mathrm{R}, \mathrm{Xu} \mathrm{X}, \mathrm{Yu} \mathrm{L}$, et al. Randomised clinical trial: gabapentin vs baclofen in the treatment of suspected refractory gastro-oesophageal reflux-induced chronic cough. Aliment Pharmacol Ther 2019;49:714-22.

10. Xu X, Lv H, Yu L, et al. A stepwise protocol for the treatment of refractory gastroesophageal reflux-induced chronic cough. J Thorac Dis 2016;8:178-85.

11. Hsu JY, Stone RA, Logan-Sinclair RB, et al. Coughing frequency in patients with persistent cough: assessment using a 24 hour ambulatory recorder. Eur Respir J 1994;7:1246-53.

12. Bai Y, Du Y, Zou D, et al. Gastroesophageal Reflux Disease Questionnaire (GerdQ) in real-world practice: a national multicenter survey on 8065 patients. J Gastroenterol Hepatol 2013;28:626-31.

13. Qiu Z, Yu L, Xu S, et al. Cough reflex sensitivity and airway inflammation in patients with chronic cough due to non-acid gastro-oesophageal reflux. Respirology 2011;16:645-52.

14. Blondeau K, Dupont LJ, Mertens V, et al. Improved diagnosis of gastro-oesophageal reflux in patients with unexplained chronic cough. Aliment Pharmacol Ther 2007;25:723-32.

15. Duan LW, Zhang S, Lin ZF. Clinical application of combined predictive factors to the diagnosis and prognosis of sepsis by logistic regression model. Chin Crit Ill Emerg Med 2017;29:139-44.
16. Meijer LL, Puik JR, Le Large TYS, et al. Unravelling the diagnostic dilemma: a microRNA panel of circulating miR-16 and miR-877 as a diagnostic classifier for distal bile duct tumors. Cancers (Basel) 2019;11:1181.

17. DeLong ER, DeLong DM, Clarke-Pearson DL. Comparing the areas under two or more correlated receiver operating characteristic curves: a nonparametric approach. Biometrics 1988;44:837-45.

18. Chung KF, Pavord ID. Prevalence, pathogenesis, and causes of chronic cough. Lancet 2008;371:1364-74.

19. French CL, Irwin RS, Curley FJ, et al. Impact of chronic cough on quality of life. Arch Intern Med 1998;158:1657-61.

20. Irwin RS, Ownbey R, Cagle PT, et al. Interpreting the histopathology of chronic cough: a prospective, controlled, comparative study. Chest 2006;130:362-70.

21. Kastelik JA, Aziz I, Ojoo JC, et al. Investigation and management of chronic cough using a probability-based algorithm. Eur Respir J 2005;25:235-43.

22. Palombini BC, Villanova CA, Araujo E, et al. A pathogenic triad in chronic cough: asthma, postnasal drip syndrome, and gastroesophageal reflux disease. Chest 1999;116:279-84.

23. Kahrilas PJ, Altman KW, Chang AB, et al. Chronic cough due to gastroesophageal reflux in adults: CHEST guideline and expert panel report. Chest 2016;150:1341-60.

24. Long L, Lai K. Characteristics of Chinese chronic cough patients. Pulm Pharmacol Ther 2019;57:101811.

25. Niimi A. Cough associated with gastro-oesophageal reflux disease (GORD): Japanese experience. Pulm Pharmacol Ther 2017;47:59-65.

26. Kinoshita Y, Kawanami C, Kishi K, et al. Helicobacter pylori independent chronological change in gastric acid secretion in the Japanese. Gut 1997;41:452-8.

27. Lai K, Chen R, Lin J, et al. A prospective, multicenter survey on causes of chronic cough in China. Chest 2013;143:613-20.

28. Yu Y, Wen S, Wang S, et al. Reflux characteristics in patients with gastroesophageal reflux-related chronic cough complicated by laryngopharyngeal reflux. Ann Transl Med 2019;7:529.

29. Kopec S, Ma J, Ferrucci J. The sensitivity and specificity of barium swallow in diagnosing gastroesophageal reflux in patients with chronic cough. Chest 2013;144:525A.

30. Liu B, Yu L, Qiu ZH, et al. The diagnostic value of multichannel intraluminal esophageal impedance and $\mathrm{pH}$ monitoring in gastroesophageal reflux-related cough. Zhonghua Nei Ke Za Zhi 2012;51:867-70. 
31. Xu X, Yang Z, Chen Q, et al. Comparison of clinical characteristics of chronic cough due to non-acid and acid gastroesophageal reflux. Clin Respir J 2015;9:196-202.

32. Morice AH, Millqvist E, Bieksiene K, et al. ERS guidelines on the diagnosis and treatment of chronic cough in adults and children. Eur Respir J 2020;5 5:1901136.

33. Sifrim D, Mittal R, Fass R, et al. Review article: acidity and volume of the refluxate in the genesis of gastrooesophageal reflux disease symptoms. Aliment Pharmacol Ther 2007;25:1003-17.

34. Agrawal A, Roberts J, Sharma N, et al. Symptoms with

Cite this article as: Wen S, Wang S, Niu S, Zhang M, Shi C, Qiu Z, Xu X, Yu L. Sensitivity and specificity of combination of Hull airway reflux questionnaire and gastroesophageal reflux disease questionnaire in identifying patients with gastroesophageal reflux-induced chronic cough. Ann Transl Med 2020;8(23):1564. doi: 10.21037/atm-20-3236 acid and nonacid reflux may be produced by different mechanisms. Dis Esophagus 2009;22:467-70.

35. Xu XH, Wang L, Liu B, et al. Clinical characteristics of non-acidic gastroesophageal reflux cough. Chin J Tubercul Res 2011;34:855-7.

36. Johansson EL, Ternesten-Hasseus E. Reliability and Validity of the Swedish Version of the Hull Airway Reflux Questionnaire (HARQ-S). Lung 2016;194:997-1005.

37. Gyawali CP, Kahrilas PJ, Savarino E, et al. Modern diagnosis of GERD: the Lyon Consensus. Gut 2018;67:1351-62. 